\title{
Design and Analysis in Multiple-Scissor-Linkage Applied to the Robotics Arm
}

\author{
Nan Hua ${ }^{1,2, a}$, Zhangong Xie ${ }^{1, b^{*}}$, Liangwei Luo ${ }^{1,2}$, Xianshuai Chen ${ }^{1}$, David J. \\ Tang $^{3}$, Hong Zhang ${ }^{2}$ \\ ${ }^{1}$ Guangzhou Institute of Advanced Technology, Chinese Academy of Sciences, Guangdong, China \\ ${ }^{2}$ Guangdong University of Technology, 100 Waihuanxi Drive, China \\ ${ }^{3}$ Smart Line Systems Inc., U.S.A. \\ anan.hua@giat.ac.cn, ${ }^{\mathrm{b}}$ zg.xie@giat.ac.cn
}

Keywords: MSL, scissor linkages, modularity, extending length, precise movement, robotics arm Abstract. Multiple-Scissor-Linkage (MSL) is kinematic mechanic, and to be a means for using two or more pairs of scissor linkages operating in different planes for moving a device in linear or nonlinear motions specifically. It is a modular component that constructs long extending, high precise movement and stronger robotic arms. It should reachmuch longer distance than conventional joint robotic arm because the multiply bar linkages like multiply arms connected one end to another.

\section{Introduction}

Robotics arm is the most welcome application in the field of robotic technology which is adopted in automation machinery equipment, military, industrial manufacturing, entertainment services, and exploration, universally, and other fields can be seen [1]. However, most of the robotics arms have limited workspace of the motion, large volume and heavy weight, sophisticated control system and so on. It intends to design the MSL to replace the upper and lower arms of conventional robots in this paper.

The MSL arm is made of aluminum alloy, so the weight is further reduced without affecting the mechanical performance. At the same time, by omitting an $\mathrm{R}-\mathrm{V}$ reducer which is mounted on the joint between the upper arm and the lower arm, and mounting one motor to the base plate, this novel robotics arm reduces the cost and the inertia which is generated during the operation. Furthermore, MSL arm has easier and more precise method than the conventional robotics arm when controlling the motion.

MSLs have abroad applications in many fields such as aviation, construction and traffic transport, et al. and drawn the attention of scholars both at home and abroad. K. K. Vu etc. proposed a new kind of deployable tension-strut structure, which is composed of a plurality of units made of bars and tether [2]. C. Gante etc. proposed a curved deployable space frame $[3,4]$. The new MSLs, composed of multiple scissor units' parallel, are spherical. On the foundation of this research, Jian S. Dai designed a complex structured ball based on mechanism decomposition and equivalent screw system analysis [5].

\section{Designation of the quadrangular MSL arm}

Structural designation of the quadrangular MSL. Planar MSL will perform satisfactorily mechanical properties when receiving the effection from the vertical force. However, it's bending strength is greatly weakened when receiving the effection from the side force. The larger extending length the planar MSL reach, the weaker the bending strength performs. Therefore, to take full advantage of the planar MSL and fully avoid its disadvantaged, this paper adopts quadrangular MSL. The quadrangular MSL can bear side force and inertial force simultaneously. Each link is connected to other links by the pin to form a planar MSL as shown in Fig.1, and four planar MSLs form a quadrangular MSL by the middle of the connection device as shown in Fig.2. In order to meet the requirements of rapid response during the movement, each pin groove are used ball bearings (It mainly 
bears radial force, and it can bear axial force, and its friction coefficient is $0.0010 \sim 0.0015$ ) to support itself and transfer movement.

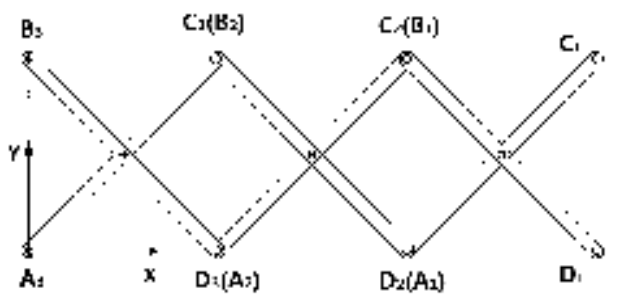

Fig. 1. Planar MSL's Structure

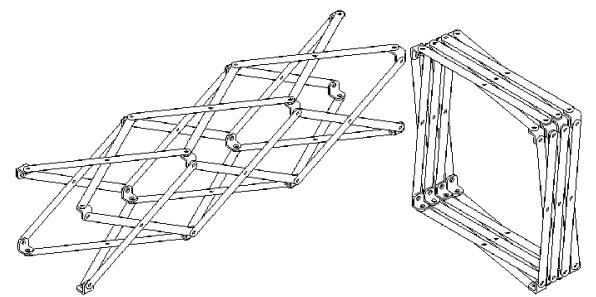

Fig.2. Quadrangular MSL's Structure

The joint of the MSL arm. There are two schemes about quadrangular MSL's transmission shown in Fig.3. Scheme one is that the mortor is mounted in point $\mathrm{D}$. That means the point $\mathrm{D}$ is fixed, while the point $\mathrm{A}, \mathrm{B}$ and $\mathrm{C}$ are moved to the point $\mathrm{D}$. Scheme two is that the mortor is mounted in the quadrilateral middle posotion. This paper decides to choose the scheme one, because it can offer larger dimension's space, and it can be easier on position control.

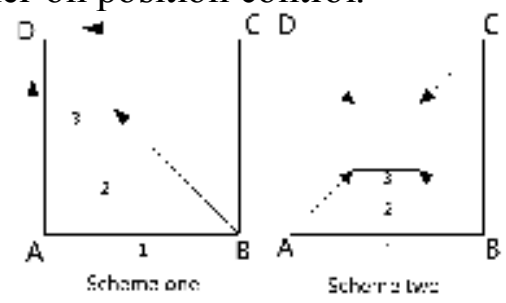

Fig.3. Transmission schemes of quadrangular MSL

In order to meet the requirements of rapid response, well vertical force performance and well side force performance, this paper adopts the linear module which is composed by ball screws and linear guide to achieve these transmission above. This paper also use $45^{\circ}$ bevel gear to transmit the servo motor power to the linear module. But we should know how to select them.

As shown in Fig.4, if we apply the external forces $\left(\mathrm{F}_{\mathrm{C}}\right.$ and $\left.\mathrm{F}_{\mathrm{D}}\right)$ in the point $\mathrm{C}$ and point $\mathrm{D}$, then we can solve the constraint forces $\left(\mathrm{F}_{\mathrm{A}}\right.$ and $\left.\mathrm{F}_{\mathrm{B}}\right)$ in the point $\mathrm{A}$ and point $\mathrm{B}$.

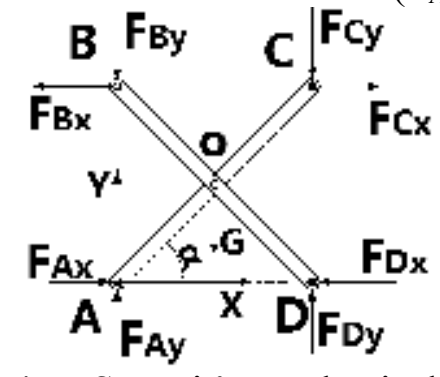

Fig.4. MSL unit's mechanical analysis

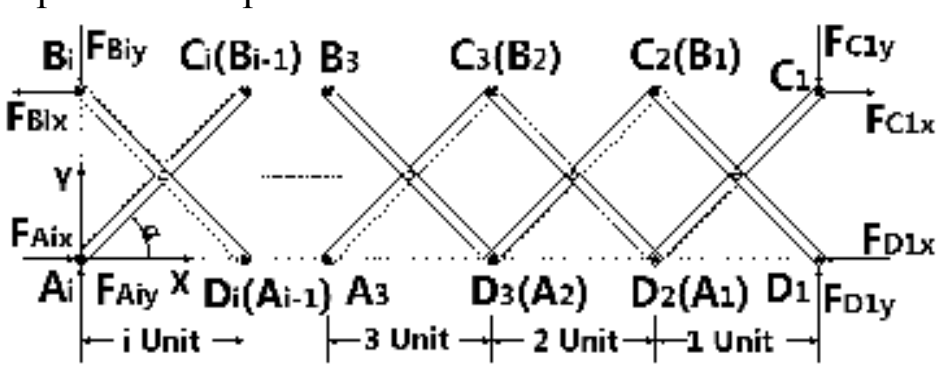

Fig.5. Planar MSL's mechanical analysis This paper establishes the following equations based on force / torque equilibrium conditions:

$$
\left[\begin{array}{ccc}
F_{D y}-F_{C y}-G / 2 & F_{B x}-F_{C x} & 0 \\
F_{D y}-F_{C y}-G / 2 & F_{A x}-F_{D x} & 0 \\
F_{B y}-F_{C y} & F_{B x}-F_{C x} & 0 \\
0 & 0 & F_{A y}+F_{D y}-G-F_{B y}-F_{C y}
\end{array}\right] \cdot\left[\begin{array}{l}
\cos \alpha \\
\sin \alpha \\
1
\end{array}\right]=0
$$

Where, $\mathrm{G}$ is one weight concerning the MSL unit. Solutions of these equations get:

$$
\begin{aligned}
& F_{A}=\left[\begin{array}{l}
F_{A x} \\
F_{A y}
\end{array}\right]=\left[\begin{array}{l}
\left(G / 2+F_{C y}-F_{D y}\right) \cdot \cot \alpha+F_{D x} \\
F_{C y}+G / 2
\end{array}\right] \\
& F_{B}=\left[\begin{array}{l}
F_{B x} \\
F_{B y}
\end{array}\right]=\left[\begin{array}{l}
\left(G / 2+F_{C y}-F_{D y}\right) \cdot \cot \alpha+F_{C x} \\
F_{D y}-G / 2
\end{array}\right]
\end{aligned}
$$

Based on it, it will solve planar MSL's constraint forces $\left(\mathrm{F}_{\mathrm{Ai}}\right.$ and $\left.\mathrm{F}_{\mathrm{Bi}}\right)$ of the i unit. As shown in Fig.5, if we apply the external forces $\left(\mathrm{F}_{\mathrm{C} 1}\right.$ and $\left.\mathrm{F}_{\mathrm{D} 1}\right)$ in the point $\mathrm{C}_{1}$ and point $\mathrm{D}_{1}$, then we can solve the constraint forces $\left(\mathrm{F}_{\mathrm{Ai}}\right.$ and $\left.\mathrm{F}_{\mathrm{Bi}}\right)$ in the point $\mathrm{A}_{\mathrm{i}}$ and point $\mathrm{B}_{\mathrm{i}}$, 
As $i$ is a odd number, that

$$
\begin{aligned}
& F_{A i}=\left[\begin{array}{l}
F_{A i x} \\
F_{A i y}
\end{array}\right]=\left[\begin{array}{l}
\left(i G / 2+F_{C 1 y}-F_{D \backslash y}\right) \cdot \cot \alpha+F_{D \backslash x} \\
F_{C 1 y}+i G / 2
\end{array}\right] \\
& F_{B i}=\left[\begin{array}{l}
F_{B i x} \\
F_{B i y}
\end{array}\right]=\left[\begin{array}{l}
\left(i G / 2+F_{C 1 y}-F_{D \backslash y}\right) \cdot \cot \alpha+F_{C \backslash x} \\
F_{D 1 y}-i G / 2
\end{array}\right]
\end{aligned}
$$

When $\mathrm{i}$ is even number, that

$$
\begin{aligned}
& F_{A i}=\left[\begin{array}{l}
F_{A i x} \\
F_{A i y}
\end{array}\right]=\left[\begin{array}{l}
(i G \cdot \cot \alpha) / 2-F_{D 1 x} \\
i G / 2-F_{D 1 y}
\end{array}\right] \\
& F_{B i}=\left[\begin{array}{l}
F_{B i x} \\
F_{B i y}
\end{array}\right]=\left[\begin{array}{l}
(i G \cdot \cot \alpha) / 2-F_{C 1 x} \\
-F_{C 1 y}-i G / 2
\end{array}\right]
\end{aligned}
$$

Simulation and analysis. As we know above, if $\alpha$ is $20^{\circ}\left(20^{\circ} \leq \alpha \leq 85^{\circ}\right)$, the MSL arm will be in a critical state. So this paper decides to establish the simulation and analysis for the quadrangular MSL and planar MSL as the $\alpha$ is $20^{\circ}$. The results of equivalent stress for quadrangular MSL are shown as Fig.6. The maximum stress value is about $51.432 \mathrm{Mpa}$ which is far less than the AL7075's allowable stress $\left([\sigma]=\sigma_{s} / n_{s}=505 / 1.3=388.462 \mathrm{Mpa}\right)$. That means the mechanical formance is satisfactory. Then, when we establish the modal simulation and analysis, we can get results and make comparison of quadrangular MSL and planar MSL as the Fig.7 shown. When the order is one, the natural frequency of planar MSL is $1.503 \mathrm{~Hz}$. However, the natural frequency of planar MSL is $9.100 \mathrm{~Hz}$ which is nearly nine times of the planar MSL. The MSL should keep away from the natural frequency above avoiding substantial structural vibration during the launched or folding process.

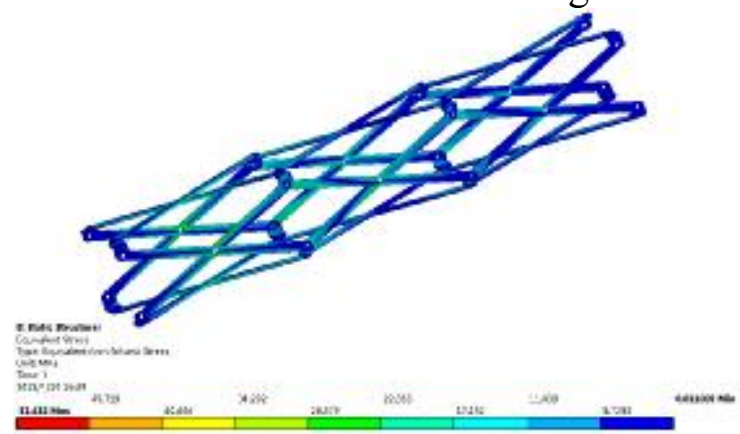

Fig.6. Stress analysis of quadrangular MSL

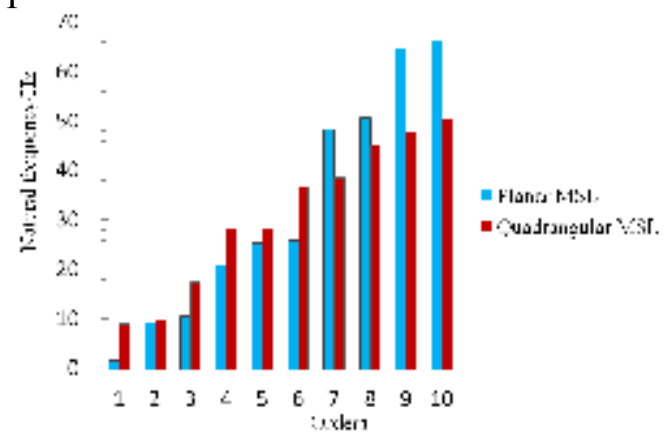

Fig.7. Comparison of modal analysis

\section{Position Control of the Arm and a Prototype}

Based on the theoretical analysis above, we can get the basic structure of the quadrangular MSL arm which is shown as the Fig. 8 . The Fig. 8 shows that the quadrangular MSL has been extended to the furthest position. Then this paper will discuss how to drive the prototype to reach the destination precisely in the way we require.

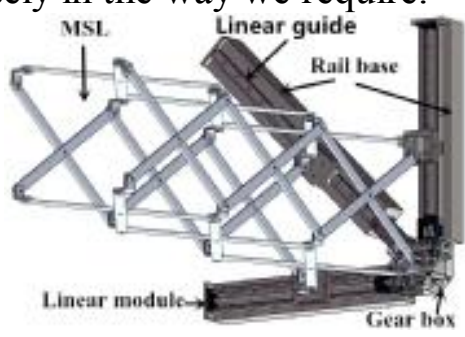

Fig. 8 The prototype of the arm

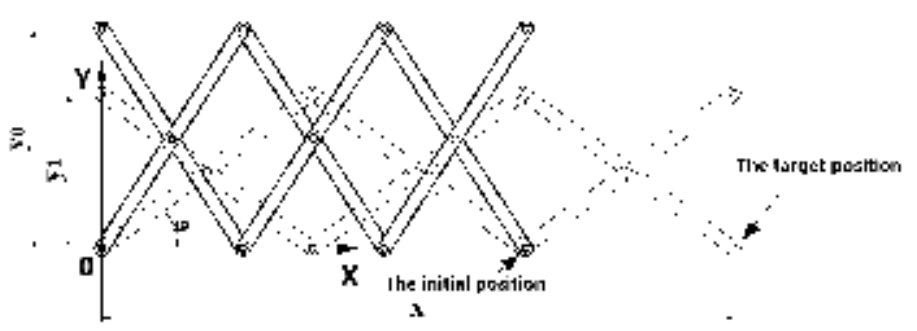

Fig. 9 The position control of the arm

As shown in Fig.9, we can get the following approximate geometrical relationship,

$$
\mathrm{x}=\mathrm{n} \cdot 1 \cdot \cos \alpha
$$


Where, $\mathrm{x}$ is the position of the target position of the arm, 1 is the length of the bar, $\alpha$ is the degree between bars and centerline of the arm, and $n$ is the number of the MSL units.

Then we can obtain the relationship between the position of $\mathrm{X}$ direction and that of $\mathrm{Y}$ direction,

$$
\mathrm{y}_{1}=(\mathrm{x} \cdot \tan \alpha) / \mathrm{n}
$$

Where, $\mathrm{y}_{1}$ is the length sensing on base by linear transducer.

As shown in Fig.8, the driving part of the arm is composed of servo motor and lead screw, which widely used in today's Computer Numeric Control (CNC) of machine tools. Lead screw can amplify the motor driving force for it divides the driving distance in many motor's turns. More importantly, lead screw can significantly improve the precisions of position control. By using motor driving lead screw, we can obtain the position of $\mathrm{Y}$ in another way,

$$
\mathrm{y}_{0}=\mathrm{ph} \cdot \mathrm{m}
$$

Where, $\mathrm{m}$ is the number of the motor turns, $\mathrm{Ph}$ is screw lead.

If obtain the accurate datas of the position of the target position of the arm, the number of the MSL units, the position of initial Y direction, the length of the bar, and the screw lead, we can easily solve the actual motor turns we input,

$$
\triangle \mathrm{m}=\left(\mathrm{y}_{0}-\mathrm{y}_{1}\right) / \mathrm{ph}=((\mathrm{ph} \cdot \mathrm{m}-\mathrm{x} \cdot \tan (\arccos (\mathrm{x} / \mathrm{n} \cdot 1)) / \mathrm{n})) / \mathrm{ph}
$$

Where, $\triangle \mathrm{m}$ is the number of the actual motor turns. When $\triangle \mathrm{m}>0$, then the motor rotates forward. When $\triangle \mathrm{m}<0$, then the motor reverses. Otherwise, the motor keeps stopping.

\section{Summary}

The novel robotics arm not only possesses satisfactory mechanical properties and simple method of position control but also perform lager range of motion than conventional robotics arm. The MSLs arm can be used as modular component to construct the systems in various applications especially in tether play-out and retrieval system and steep terrain adherence for vertical and horizontal mobility.

\section{Acknowledgements}

This work is supported by the Guangzhou major project funding: The research and development of new industrial robotics arms based on high-manufacturing industries (2014J4500029).

\section{References}

[1]. John J. Craig, Introduction to Robotics, Mechanics \& Control, Addison-Wesley Publishing Company.

[2]. Vu, K. K., Liew, J. Y. R., Anandasivam, K. Deployable Tension-Strut Structures: from Concept to implementation [J]. Journal of Constructional Steel Research, 2006. 62: 195-209.

[3]. C. Gantes, J., Connor, J., Logcher, R.D., Combining Numerical Analysis and Engineering Judgment to Design Deployable Structures [R]. 1991, Massachusetts Institute of Technology: Cambridge,. P. 431-440.

[4]. C. Gantes, An Improved Analytical Model for the Prediction of the Nonlinear Behavior of Flat and Curved Deployable Space Frames [R]. 1997, Department of Civil Engineering: Athens. P. 129-158.

[5]. Dai, J.S., Li, D., Zhang, Q., Jin, G. Mobility Analysis of a Complex Structured Ball Based on Mechanism Decomposition and Equivalent Screw System Analysis [J]. 